\title{
INEQUALITIES FOR THE SCHATTEN $p$-NORM II
}

\author{
by FUAD KITTANEH
}

(Received 15 August, 1985)

This paper is a continuation of [3] in which some inequalities for the Schatten $p$-norm were considered. The purpose of the present paper is to improve some inequalities in [3] as well as to give more inequalities in the same spirit.

Let $H$ be a separable, infinite dimensional complex Hilbert space, and let $B(H)$ denote the algebra of all bounded linear operators acting on $H$. Let $K(H)$ denote the closed two-sided ideal of compact operators on $H$. For any compact operator $A$, let $|A|=\left(A^{*} A\right)^{1 / 2}$ and $s_{1}(A), s_{2}(A), \ldots$ be the eigenvalues of $|A|$ in decreasing order and repeated according to multiplicity. A compact operator $A$ is said to be in the Schatten $p$-class $C_{p}(1 \leqslant p<\infty)$, if $\sum s_{i}(A)^{p}<\infty$. The Schatten $p$-norm of $A$ is defined by $\|A\|_{p}=\left(\sum s_{i}(A)^{p}\right)^{1 / p}$. This norm makes $C_{p}$ into a Banach space. Hence $C_{1}$ is the trace class and $C_{2}$ is the Hilbert-Schmidt class. It is reasonable to let $C_{\infty}$ denote the ideal of compact operators $K(H)$, and $\|\cdot\|_{\infty}$ stand for the usual operator norm.

If $A \in C_{1}$ and $\left\{e_{i}\right\}$ is an orthonormal basis of $H$, then the trace of $A$, denoted by tr $A=\sum\left(A e_{i}, e_{i}\right)$ is independent of the choice of $\left\{e_{i}\right\}$. If $A \in C_{p}$ and $B \in C_{q}$, then $A B \in C_{1}$, $\operatorname{tr}(A B)=\operatorname{tr}(B A)$, and $|\operatorname{tr}(A B)| \leqslant\|A\|_{p}\|B\|_{q}$ whenever $1 / p+1 / q=1$. If $\left\{e_{i}\right\}$ is any orthonormal set in $H$, then for $A \in C_{p},\|A\|_{p}^{p} \geqslant \Sigma\left|\left(A e_{i}, e_{i}\right)\right|^{p}$. The reader is referred to [4] or [5] for further properties of the Schatten $p$-classes.

In [6], G. Weiss proved that if $N$ is a normal operator in $B(H)$ and if $X \in C_{2}$ is such that $N X-X N \in C_{1}$, then $\operatorname{tr}(N X-X N)=0$. This result admits the following extension.

Theorem 1. If $N$ is normal in $B(H), X \in C_{2}$, and $A \in B(H)$ is such that $A X-X N \in$ $C_{1}$, then $|\operatorname{tr}(A X-X N)| \leqslant\|X\|_{2}\|A-N\|_{2}$.

Proof. If $A-N$ is not in $C_{2}$, then the result is trivial. We therefore assume that $A-N \in C_{2}$. Thus $(A-N) X \in C_{1}$ and so $A X-X N=N X-X N+(A-N) X$ implies that $N X-X N \in C_{1}$. Now Weiss's result implies that $\operatorname{tr}(N X-X N)=0$. Therefore, $\operatorname{tr}(A X-X N)=\operatorname{tr}((N-A) X)$ from which it follows that $|\operatorname{tr}(A X-X N)| \leqslant\|X\|_{2}\|A-N\|_{2}$.

If $H$ is finite dimensional, then every commutator, that is an operator of the form $A X-X A$, has zero trace. In fact, it is well-known $[1$, p. 128] that an operator on a finite dimensional Hilbert space is a commutator if and only if it has trace 0 . Thus if $A, B$, and $X$ are operators on $H$ with $\operatorname{dim} H=n$, then $A X-X B=A X-X A+X(A-B)$. Since $\operatorname{tr}(A X-X A)=0$, it follows that $|\operatorname{tr}(A X-X B)| \leqslant\|X\|_{q}\|A-B\|_{p}$ whenever $1 / p+1 / q$ $=1$. In particular if $X$ is the identity operator, then $|\operatorname{tr}(A-B)| \leqslant n^{1 / q}\|A-B\|_{p}$ which is known. This inequality may be useful in approximation problems of operators on a finite dimensional Hilbert space. For, if $C$ is an operator with $\operatorname{tr} C=0$, then for any operator $A$ we have $|\operatorname{tr} A|=|\operatorname{tr}(A-C)| \leqslant n^{1 / q}\|A-C\|_{p}$ and so $\|A-C\|_{p} \geqslant \frac{|\operatorname{tr} A|}{n^{1 / q}}$. But if we choose 
$C=A-\frac{\operatorname{tr} A}{n}$, and hence $\operatorname{tr} C=0$, then

$$
\|A-C\|_{p}=\left\|\frac{\operatorname{tr} A}{n}\right\|_{p}=\frac{|\operatorname{tr} A|}{n} n^{1 / p}=\frac{|\operatorname{tr} A|}{n^{1 / q}} .
$$

Thus we have proved the following result.

THEOREM 2. If $\operatorname{dim} H=n$ and $A \in B(H)$, then $\min \left\{\|A-C\|_{p}: \operatorname{tr} C=0\right\}=\frac{|\operatorname{tr} A|}{n^{1 / q}}$ whenever $1 / p+1 / q=1$.

Theorem 2 can be formulated in terms of commutators to yield the following inequality.

Corollary 1. If $\operatorname{dim} H=n$, then for any operators $A, B$, and $X$ acting on $H$, we have $\|A+B X-X B\|_{p} \geqslant \frac{|\operatorname{tr} A|}{n^{1 / q}}$ whenever $1 / p+1 / q=1$.

It has been shown in [3] that if $A$ is an operator in $B(H)$ such that $\operatorname{Im} A \geqslant a \geqslant 0$, then for any $X \in B(H),\left\|A X-X A^{*}\right\|_{p} \geqslant a\|X\|_{p}(1 \leqslant p \leqslant \infty)$. Replacing $A$ by $i A$, this inequality becomes $\left\|A X+X A^{*}\right\|_{p} \geqslant a\|X\|_{p}$ whenever $\operatorname{Re} A \geqslant a \geqslant 0$. The remarkable Clarkson-McCarthy inequalities [5] can be used to improve Theorem 3 in [3] for $1<p<\infty$ as follows.

THEOREM 3. If $A$ is an operator in $B(H)$ such that $\operatorname{Im} A \geqslant a \geqslant 0$, then for any $X \in B(H)$

and

$$
\left\|A X-X A^{*}\right\|_{p} \geqslant\left(4^{1 / p} a\right)\|X\|_{p} \quad(2 \leqslant p \leqslant \infty)
$$

$$
\left\|A X-X A^{*}\right\|_{p} \geqslant\left(4^{1 / q} a\right)\|X\|_{p} \quad(1 \leqslant p \leqslant 2),
$$

where $1 / p+1 / q=1$. In particular, $\left\|A X-X A^{*}\right\|_{2} \geqslant(2 a)\|X\|_{2}$.

Proof. If $T=\operatorname{Re} T+i \operatorname{Im} T$ is the cartesian decomposition of an operator $T$ in $B(H)$, then it is not hard to conclude from the Clarkson-McCarthy inequalities that

$$
\|\operatorname{Re} T\|_{p}^{p}+\|\operatorname{Im} T\|_{p}^{p} \leqslant\|T\|_{p}^{p} \leqslant \frac{2^{p}}{4}\left(\|\operatorname{Re} T\|_{p}^{p}+\|\operatorname{Im} T\|_{p}^{p}\right) \quad(2 \leqslant p<\infty)
$$

and

$$
\frac{2^{p}}{4}\left(\|\operatorname{Re} T\|_{p}^{p}+\|\operatorname{Im} T\|_{p}^{p}\right) \leqslant\|T\|_{p}^{p} \leqslant\|\operatorname{Re} T\|_{p}^{p}+\|\operatorname{Im} T\|_{p}^{p} \quad(1<p \leqslant 2) .
$$

Let $X=Y+i Z$ be the cartesian decomposition of $X$, then $A Y-Y A^{*}=i \operatorname{Im}\left(A X-X A^{*}\right)$ and $A Z-Z A^{*}=-i \operatorname{Re}\left(A X-X A^{*}\right)$. But, as in the proof of Theorem 3 in [3], we have $\left\|A Y-Y A^{*}\right\|_{p} \geqslant(2 a)\|Y\|_{p}$ and $\left\|A Z-Z A^{*}\right\|_{p} \geqslant(2 a)\|Z\|_{p}$ for all $1 \leqslant p \leqslant \infty$. Now if 
$2 \leqslant p<\infty$, then

$$
\begin{aligned}
\left\|A X-X A^{*}\right\|_{p}^{p} & \geqslant\left\|A Y-Y A^{*}\right\|_{p}^{p}+\left\|A Z-Z A^{*}\right\|_{p}^{p} \\
& \geqslant(2 a)^{p}\left(\|Y\|_{p}^{p}+\|Z\|_{p}^{p}\right) \\
& \geqslant(2 a)^{p}\left(\frac{4}{2^{p}}\right)\|X\|_{p}^{p} .
\end{aligned}
$$

Hence $\left\|A X-X A^{*}\right\|_{p} \geqslant\left(4^{1 / p} a\right)\|X\|_{p}$.

If $1<p \leqslant 2$, then

$$
\begin{aligned}
\left\|A X-X A^{*}\right\|_{p}^{p} & \geqslant \frac{2^{p}}{4}\left(\left\|A Y-Y A^{*}\right\|_{p}^{p}+\left\|A Z-Z A^{*}\right\|_{p}^{p}\right) \\
& \geqslant\left(\frac{2^{p}}{4}\right)(2 a)^{p}\left(\|Y\|_{p}^{p}+\|Z\|_{p}^{p}\right) \\
& \geqslant\left(\frac{2^{p}}{4}\right)(2 a)^{p}\|X\|_{p}^{p} .
\end{aligned}
$$

Hence

$$
\left\|A X-X A^{*}\right\|_{p} \geqslant \frac{4 a}{4^{1 / p}}\|X\|_{p}
$$

and so

$$
\left\|A X-X A^{*}\right\|_{p} \geqslant\left(4^{1 / q} a\right)\|X\|_{p}
$$

where $1 / p+1 / q=1$. lemma.

Employing Berberian's trick also enables us to generalize Theorem 3. First we need a

Lemma. Let $X$ be an operator in $B(H)$. If $Y=\left[\begin{array}{ll}0 & X \\ 0 & 0\end{array}\right]$ is an operator defined on $H \oplus H$, then $\|Y\|_{p}=\|X\|_{p}$ for $1 \leqslant p \leqslant \infty$.

Proof. It is clear that $X \in C_{p}$ on $H$ if and only if $Y \in C_{p}$ on $H \oplus H$. The desired conclusion now follows from the observation that $Y^{*} Y=\left[\begin{array}{cc}0 & 0 \\ 0 & X^{*} X\end{array}\right]$ or equivalently $|Y|=\left[\begin{array}{cc}0 & 0 \\ 0 & |X|\end{array}\right]$.

THEOREM 4. If $A$ and $B$ are operators in $B(H)$ such that $\operatorname{Im} A \geqslant a \geqslant 0$ and $\operatorname{Im} B \geqslant b \geqslant 0$, then for any $X \in B(H)$,

and

$$
\left\|A X-X B^{*}\right\|_{p} \geqslant\left(4^{1 / p} \min (a, b)\right)\|X\|_{p} \quad(2 \leqslant p \leqslant \infty)
$$

$$
\left\|A X-X B^{*}\right\|_{p} \geqslant\left(4^{1 / q} \min (a, b)\right)\|X\|_{p} \quad(1 \leqslant p \leqslant 2),
$$

where $1 / p+1 / q=1$.

Proof. On $H \oplus H$, let $T=\left[\begin{array}{cc}A & 0 \\ 0 & B\end{array}\right]$ and let $Y=\left[\begin{array}{ll}0 & X \\ 0 & 0\end{array}\right]$. Then $\operatorname{Im} T \geqslant \min (a, b) \geqslant 0$ 
and $T Y-Y T^{*}=\left[\begin{array}{cc}0 & A X-X B^{*} \\ 0 & 0\end{array}\right]$. The result now follows by applying the lemma and Theorem 3 to the operators $T$ and $Y$.

It should be noticed that if $B^{*}$ is replaced by $B$ in Theorem 4 , then the result is no longer true.

Whether Theorem 3, and hence its generalization, Theorem 4, can be improved further so that $\left\|A X-X A^{*}\right\|_{p} \geqslant(2 a)\|X\|_{p}$ for all $1 \leqslant p \leqslant \infty$ is not known to the author. This result is obtained when either $p=2$ or $X$ is taken to be self-adjoint or more generally seminormal ( $X$ or $X^{*}$ is hyponormal). As an application of the last assertion we now obtain Proposition 3.2 in [2] and extend it so that it includes the case $p=\infty$ as well.

THEOREM 5. If $A$ and $B$ are self-adjoint operators in $B(H)$ and $A+B \geqslant a \geqslant 0$, then $\left\|A^{2}-B^{2}\right\|_{p} \geqslant a\|A-B\|_{p}$ for $1 \leqslant p \leqslant \infty$.

Proof. Let $S=A-B$ and $T=i(A+B)$. Then $\operatorname{Im} T \geqslant a \geqslant 0, S$ is self-adjoint, and $T S-S T^{*}=2 i\left(A^{2}-B^{2}\right)$. The result now follows by appealing to the inequality $\left\|T S-S T^{*}\right\|_{p} \geqslant(2 a)\|S\|_{p}$.

The case $p=\infty$ is of particular importance. It asserts that the square root function is continuous on the interior of the positive cone of $B(H)$. A similar remark has been made about $C_{1}$ in [2]. It also follows from Theorem 5 that if $A$ and $B$ are self-adjoint operators in $B(H)$ such that $A^{2}=B^{2}$ and $A+B \geqslant a>0$, then $A=B$. Of course these equality signs may be taken modulo $C_{p}$. For general operators $A$ and $B$ in $B(H)$, it may be that $A^{2}=B^{2}$ and $A^{3}=B^{3}$, yet $A \neq B$. For example, take $A$ and $B$ to be distinct nilpotent operators of index two. The following theorem is a positive result in this direction.

THEOREM 6. Let $A$ and $B$ be operators in $B(H)$ such that $A^{2}=B^{2}$ and $A^{3}=B^{3}$. If $\operatorname{ker} A \subset \operatorname{ker} A^{*}$ and $\operatorname{ker} B \subset \operatorname{ker} B^{*}(\operatorname{ker} A$ denotes the kernel of $A)$, then $A=B$.

Proof. Let $C=A-B$. Now $A^{2} C=A^{3}-A^{2} B=A^{3}-B^{3}=0$. Thus from the assumption that $\operatorname{ker} A \subset \operatorname{ker} A^{*}$, it follows that $A^{*} A C=0$. Hence $(A C)^{*}(A C)=0$ and so $A C=0$. Thus $A^{*} C=0$. Similarly $B^{2} C=0$ and $\operatorname{ker} B \subset \operatorname{ker} B^{*}$ imply that $B C=0$ and $B^{*} C=0$. Therefore $C^{*} C=\left(A^{*}-B^{*}\right) C=A^{*} C-B^{*} C=0$. Whence $C=0$ and so $A=B$ as required.

Remarks. 1. Algebraic manipulations and induction show that the powers 2 and 3 in Theorem 6 can be replaced by any two relatively prime powers $n$ and $m$.

2. The following two conditions are important special cases of the kernel assumption given in Theorem 6: (a) $A$ is one-to-one and $B$ is one-to-one; (b) $A$ and $B$ are hyponormal operators.

Next we establish the following inequality, the proof of which has a flavor similar to that of Theorems 2 and 3 in [3].

Theorem 7. If $A$ and $B$ are operators in $B(H)$ such that $A+B \geqslant a \geqslant 0$, then for any seminormal operator $X$ in $B(H),\left\|X A X^{*}+X^{*} B X\right\|_{p} \geqslant a\|X\|_{2 p}^{2}$ for $1 \leqslant p \leqslant \infty$. 
Proof. We consider two cases.

Case 1. $p=\infty$. Without loss of generality we may assume that $X$ is hyponormal. Hence there exists a sequence $\left\{f_{n}\right\}$ of unit vectors in $H$ such that $(X-t) f_{n} \rightarrow 0$ as $n \rightarrow \infty$ where $|t|=\|X\|$.

Since $X-t$ is also hyponormal, it follows that $(X-t)^{*} f_{n} \rightarrow 0$ as $n \rightarrow \infty$. Now

$$
\begin{aligned}
\left\|X A X^{*}+X^{*} B X\right\| \geqslant & \left|\left(\left(X A X^{*}+X^{*} B X\right) f_{n}, f_{n}\right)\right| \\
= & \left.\left|\left(A X^{*} f_{n},(X-t)^{*} f_{n}\right)+t\left(A(X-t)^{*} f_{n}, f_{n}\right)+\right| t\right|^{2}\left(A f_{n}, f_{n}\right) \\
& +\left(B X f_{n},(X-t) f_{n}\right)+\bar{t}\left(B(X-t) f_{n}, f_{n}\right)+|t|^{2}\left(B f_{n}, f_{n}\right) \mid
\end{aligned}
$$

$\geqslant a|t|^{2}$ minus a term which goes to zero as $n \rightarrow \infty$. Hence $\left\|X A X^{*}+X^{*} B X\right\| \geqslant$ $a\|X\|^{2}$ as required.

Case 2. $1 \leqslant p<\infty$. There is nothing to prove if $X A X^{*}+X^{*} B X$ is not in $C_{p}$. We therefore assume that $X A X^{*}+X^{*} B X \in C_{p}$, and hence it is in particular compact. If $\pi: B(H) \rightarrow B(H) / C_{\infty}$ is the quotient map of $B(H)$ onto the Calkin algebra $B(H) / C_{\infty}$, then we have $\pi(X) \pi(A) \pi(X)^{*}+\pi(X)^{*} \pi(B) \pi(X)=0$. Applying case 1 now implies that $\pi(X)=0$, whence $X$ is compact. But is is known $[1$, p. 110] that a compact hyponormal operator is diagonalizable, and hence $X e_{n}=t_{n} e_{n}$ where $\left\{e_{n}\right\}$ is an orthonormal basis of $H$. Thus

$$
\begin{aligned}
\left\|X A X^{*}+X^{*} B X\right\|_{p}^{p} & \geqslant \sum\left|\left(\left(X A X^{*}+X^{*} B X\right) e_{n}, e_{n}\right)\right|^{p} \\
& =\sum\left|\left(A X^{*} e_{n}, X^{*} e_{n}\right)+\left(B X e_{n}, X e_{n}\right)\right|^{p} \\
& =\left.\left.\sum|| t_{n}\right|^{2}\left((A+B) e_{n}, e_{n}\right)\right|^{p} \\
& \geqslant a^{p} \sum\left|t_{n}\right|^{2 p} \\
& =a^{p}\|X\|_{2 p}^{2 p} .
\end{aligned}
$$

Therefore $\left\|X A X^{*}+X^{*} B X\right\|_{p} \geqslant a\|X\|_{2 p}^{2}$.

We point out here that Theorem 7 is not true if the semi-normality assumption on $X$ is removed. For example, consider

$$
X=\left[\begin{array}{ll}
0 & 1 \\
0 & 0
\end{array}\right], \quad A=\left[\begin{array}{ll}
1 & 0 \\
0 & 0
\end{array}\right], \quad \text { and } B=\left[\begin{array}{ll}
0 & 0 \\
0 & 1
\end{array}\right],
$$

which act on a two-dimensional Hilbert space. Also if $X$ is hyponormal and $A+B \geqslant a \geqslant 0$, then it need not be true that $X A X^{*}+X^{*} B X \geqslant a X X^{*}$. For example, let $X=U$, the unilateral shift operator, $A=U U^{*}$, and $B=1-U U^{*}$. Then $X A X^{*}+X^{*} B X=U^{2} U^{* 2}$ and $a X X^{*}=U U^{*}$. The assertion now follows since $U$ is a nonunitary isometry.

Finally, we state the following theorem. The proof is omitted since it is similar to that of Theorem 7. 
TheOREM 8. If $A$ and $B$ are operators in $B(H)$ such that $A+B \geqslant a \geqslant 0$, then for any seminormal operator $X$ in $B(H),\|A X+X B\|_{p} \geqslant a\|X\|_{p}$ for $1 \leqslant p \leqslant \infty$.

If $A$ and $B$ are self-adjoint operators in $B(H)$ with $A+B \geqslant a \geqslant 0$, and $X=A-B$, then $A X+X B=A^{2}-B^{2}$. Hence Theorem 5 is obtained as a special case of Theorem 8 .

ADDED IN Proof. It has been shown recently by the author in Inequalities for the Schatten $p$-norm III, Comm. Math. Phys. 104 (1986), 307-310 that if $A$ is an operator in $B(H)$ such that $\operatorname{Im} A \geqslant a \geqslant 0$, then for any $X$ in $B(H)$, we have

$$
\left\|A X-X A^{*}\right\|_{p} \geqslant 2 a\|X\|_{p}(1 \leqslant p \leqslant \infty),
$$

which is the desired improvement of Theorem 3.

\section{REFERENCES}

1. P. R. Halmos, A Hilbert space problem book (Springer-Verlag, 1982).

2. J. L. van Hemmen and T. Ando, An inequality for trace ideals, Comm. Math. Phys. 76 (1980), 143-148.

3. F. Kittaneh, Inequalities for the Schatten p-norm, Glasgow Math. J. 26 (1985), 141-143.

4. J. R. Ringrose, Compact non-self-adjoint operators (Van Nostrand Reinhold Co., 1971).

5. B. Simon, Trace ideals and their applications (Cambridge University Press, 1979).

6. G. Weiss, The Fuglede commutativity theorem modulo the Hilbert-Schmidt class and generating functions for matrix operators. I, Trans. Amer. Math. Soc. 246 (1978), 193-209.

Department of Mathematics

UnITED ARAB EMIRATES UNIVERSITY

P.O. BOX 15551

AL-AIN, U.A.E. 